$$
\text { "klukovits" — 2005/2/17 — 18:26 — page } 397 \text { — \#1 }
$$

\title{
The first clear distinction between the heuristic conjecture and the deductive proof in the ancient mathematics
}

\author{
LAJOS KLUKOVITS
}

Abstract. The mathematics of the ancient river-valley cultures was purely empirical, while the classical Greek mathematics was entirely deductive without any written sign of the heuristic arguments. In the forthcoming Hellenistic period there were significant changes. One of them is that in spite of the rigorous (deductive) proofs some heuristic arguments appeared in separate treatises. We show a nice example due to Archimedes.

We have learned from the very pioneers of this science not to have regard to mere plausible imaginings when it is a question of the reasonings to be included in our geometrical doctrine. Proclus

Key words and phrases: heuristic arguments, deductive proof.

ZDM Subject Classification: A39, D59.

1. A pearl of the Mesopotamian mathematics from the period of the Old Babylonian Empire

It is well-known that the mathematics of the ancient river-valley cultures was purely empirical. We can find in the texts concrete numerical problems and their solutions only. They did not compose general rules, general procedures or even precise definitions. However, in almost all cases the solutions are formulated like general features, serving as a recipe for similar numerical problems. As an

Copyright (C) 2004 by University of Debrecen 


$$
\text { "klukovits" — 2005/2/17 — 18:26 — page 398 — \#2 }
$$

example we show a problem leading to a quadratic equation using the description introduced by Neugebauer $[4,5]$ for hexagesimal numbers.

Problem (in BM13901 [4]). Find the side of a square, if the area less the side is 14,30 .

The original solution on a clay-tablet reads as follows:

Take half of 1 , which is $0 ; 30$, and multiply $0 ; 30$ by $0 ; 30$, which is $0 ; 15$.

Add this to 14,30 to get 14,$30 ; 15$. This is the square of $29 ; 30$. Now add

$0 ; 30$ to $29 ; 30$, and the result is 30 , the side of the square.

In modern terminology we have to solve the quadratic equation

$$
x^{2}-a x=b, \quad \text { where } a=1, b=14 ; 30,
$$

and the original solution can be formulated as

$$
x=\sqrt{\left(\frac{a}{2}\right)^{2}+b}+\frac{a}{2},
$$

i.e. we can find out the well-known formula behind the calculation.

We have to emphasis that the Mesopotamian scribes had not used formulas. His train of thought was simply step-by-step calculations in a routine way.

\section{Selected items from the classical Greek mathematics}

The mathematics of the Greek classical period is totally different. In the brilliant collection, in the Elements of Euclid [2] - roughly speaking - we can find numbers as serial numbers only, but sequences of postulates, axioms, definitions and (theoretical) propositions followed by rigorous proofs based on the postulates and previous propositions. As an illustration we show some definitions and propositions, using the text of [2].

Definitions from Book VII.

1. An unit is that by virtue of which each things that exist is called one.

2. A number is a multitude composed of units.

3. A number is a part of a number, the less of the greater, when it measures the greater.

11. A prime number is that which is measured by an unit alone. 


$$
\text { "klukovits" — 2005/2/17 — 18:26 — page 399 — \#3 }
$$

Proposition IX. 20. Prime numbers are more than any assigned multitude of prime numbers.

The proof is the same as in any modern textbook on number theory with the tiny difference that for Euclid finite number means 3 (essentially $A, B, C$ ) only, instead of writing $p_{1}, \ldots, p_{k}$.

Proposition X. 1. Two unequal magnitudes being set out, if from the greater there be substracted a magnitude greater than its half, and from that which is left a magnitude greater than its half, and is this process be repeated continually, there will be left some magnitude which will be less than the lesser magnitude set out.

Proposition XII. 2. Circles are one to another as the squares on the diameters.

It is easy to observe that we do not find not only calculations but even any heuristic argument. We do not read on the way of discovering the propositions or even on the background of the definitions, postulates. The mathematics of this time is purely deductive without any empirism.

\section{Some features of the Greek mathematics in the Hellenistic period}

The earlier feature of the mathematics was somewhat changed in the forthcoming Hellenistic time. Firstly, the less important from our point of view, that we can find calculations besides the deductively proved theorems. As an example we mention that Euclid was interested in the area of a circle such as written in the above mentioned Proposition XII. 2 only, while Archimedes calculated the ratio of the area of two circle, namely he stated that this ratio - denoted by $\pi-$ satisfies the inequalities

$$
\frac{21}{7}<\pi<\frac{223}{71}
$$

The second, the really important in our extent, is that in this time certain mathematicians not only establish propositions with rigorous proofs but sometimes write down the way of finding their results. In modern terminology they publish the heuristic arguments by which they formulate the statements to be proved. The pioneer of this approach to mathematics was Archimedes. First consider a part of a letter to ERATOSTHEnEs [1]. 
"klukovits" — 2005/2/17 — 18:26 — page 400 — \#4

Archimedes to Eratosthenes greeting!

On an earlier occasion I sent you some of the theorems found by me, the propositions of which I have written down, urging you to find the proofs which I did not yet communicate at the time.

This is followed by two theorems on a cylinder inscribed in a right prism having a square for its base and on a cylinder inscribed in a cube.

I will sent you the proofs of these theorems in this book.

Since as I said, I know that you are diligent, an excellent teacher of philosophy, and greatly interested in any mathematical investigations that may come your way, I thought it might be appropriate to write down and set forth for you in the same book a certain special method, by means of which you will be enable to recognize certain mathematical questions with the aid of mechanics. I am convinced that this is no less useful for finding the proofs of these same theorems. For some things, which first became clear to me by a mechanical method, were afterwards proven geometrically, because their investigation by the said method does not furnish an actual demonstration.

He wrote a series of such letters from Syracusa to Alexandria. Usually in a letter he wrote some statement derived by mechanical way and asked his partner - mostly Eratosthenes - to prove them geometrically. Afterwards in a separate letter the deductive proof was sent also.

Unfortunately the tractat(s) with his heuristic argumentations - mostly based on his results on mechanics - was(were) not known until the beginnings of the $20^{\text {th }}$ century. This was the main reason why most historians of mathematics drew less attention to the heuristic part of the Archimedian mathematics. But the scene changed after 1906.

At the beginnig of the $20^{\text {th }}$ century J. L. Heiberg found a report by Papadopulos Cerameus on a palimpset with originally mathematical content in the library of the monastry of the Holy Sepulchre at Jerusalem. He examined this manuscript at Constantinople in the years 1906 and 1908. It proved to contain an Archimedes text of the $10^{\text {th }}$ century written on parchment, which had been tried to efface in the the $12-14^{\text {th }}$ centuries, in order to write a Euchologium in its stead.

Heiberg was succesful in reconstructing the original text proved to be Archimedian. It contains fragments of several Archimedian treatises, e.g. On the Sphere and the Cylinder, Measurement of the Circle, On the Equilibrium of Planets (all known from other sources). Among them we can read in it two very important 
treatises. One is a considerable part of the Greek text of On Floating Bodies, and the second - the more important - an almost complete text of an as yet unknown work of Archimedes, the existence of which was known from ancient quotations and commentaries. It was referred as $E \varphi o \delta o \zeta$, which can be translated as METHOD.

This treatise starts with several lemmas on centres of gravity, some of which are originally stated as postulates or propositions in On the Equilibrium of Planets. These are followed by the statement very important in our point of view [1].

Proposition. Let the segment $\alpha \beta \gamma$ be given, comprehended by the straight line $\alpha \gamma$ and the orthotome (parabola) $\alpha \beta \gamma$; let $\alpha \gamma$ be bisected in $\delta$, let $\delta \beta \varepsilon$ be drawn parallel to the diameter, and let $\beta \alpha$ and $\beta \gamma$ be joined.

I say that the segment $\alpha \beta \gamma$ is larger by one-third than the triangle $\alpha \beta \gamma$.

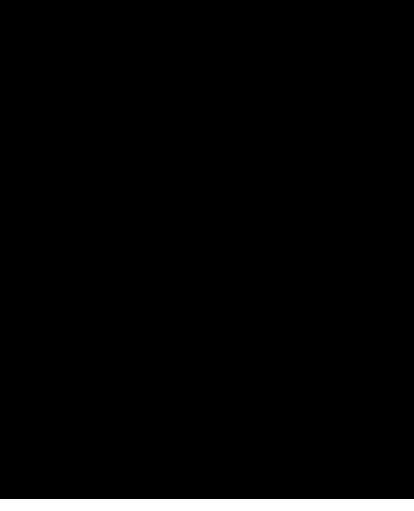

Figure 1

\section{The Construction.}

$\alpha \beta \gamma \quad$ the parabola segment bounded by the straight line $\alpha \gamma$ and the arc $\alpha \beta \gamma$,

$\gamma \zeta$ tangent to the parabola at $\gamma$,

$\delta \quad$ the midpoint of $\alpha \gamma$,

$\delta \beta \varepsilon \quad$ the diameter throught $\delta$ (a line parallel to the axis),

$\xi \quad$ a variable point on the line $\alpha \gamma$,

$\xi o \nu \mu$ a line parallel to $\delta \varepsilon$,

$\alpha \kappa \zeta \quad$ a line parallel to $\delta \varepsilon$,

$\kappa \vartheta=\kappa \gamma$. 


$$
\text { "klukovits" — 2005/2/17 — 18:26 — page } 402 \text { — \#6 }
$$

By Proposition 2.2 of the tractat On Conoid and Spheroid (also contained in the lost Conics of Euclid)

$$
\delta \beta=\beta \varepsilon
$$

thus

$$
\alpha \kappa=\kappa \zeta \quad \text { and } \quad \xi \nu=\nu \mu .
$$

The Heuristic Arguments [1, 3].

Compare the area of the parabola segment and the area of the triangle $\alpha \gamma \zeta$ on the basis of the following notions:

- the area of a figure: the sum of weighted (parallel) line segments covering the figure,

- the weight of a line segment: the length of it,

- the area of the parabola segment: the sum of weighted line segments such as $\xi o$,

- the area of the triangle $\alpha \gamma \zeta$ : the sum of weighted line segments such as $\xi \mu$.

Then Archimedes proved that

$$
\vartheta \kappa \cdot \xi o=\kappa \nu \cdot \xi \mu \text {. }
$$

The mechanical analysis of this equation:

( 2 ) Consider $\vartheta \kappa$ and $\kappa \nu$ as arms of a lever with fulcrum at $\kappa$.

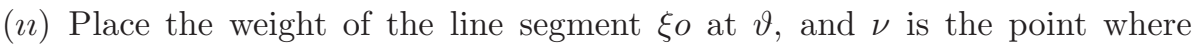
placing the weight of the line segment $\xi \mu$ we reach equilibrium position.

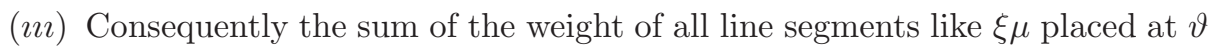
will balance the sum of all line segments like $\xi \mu$ their weight placed at its midpoint.

$(\imath \nu)$ The collection of line all line segments like $\xi \mu$ the weight of each placed at its midpoint (the center of gravity) is "equivalent" to the triangle $\alpha \gamma \zeta$ placed at its center of gravity.

$(\nu)$ Using a result from the book On the Equilibrium os Planets the above center of gravity is at point $\chi$ on $\gamma \kappa$ and

$$
\kappa \chi=\frac{1}{3} \gamma \kappa
$$


$(\nu \imath)$ By the law of the lever

$$
\kappa \chi \cdot \text { area } \alpha \gamma \zeta_{\triangle}=\vartheta \kappa \cdot \text { area of the segment } \alpha \beta \gamma,
$$

thus

$$
\frac{\alpha \gamma \zeta_{\triangle}}{\operatorname{segment} \alpha \beta \gamma}=\frac{\kappa \vartheta}{\kappa \chi}=\frac{3}{1} .
$$

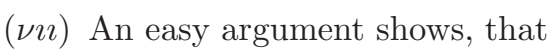

$$
\frac{\text { segment } \alpha \beta \gamma}{\alpha \gamma \beta_{\triangle}}=\frac{4}{3} .
$$

Archimedes emphasized that the above argument is not a mathematical proof, a heuristical conjecture only, that should be proved geometrically.

\section{The Deductive Proof.}

The (deductive) proof of Archimedes is based on a theorem of Euclid (Proposition X.1. in the Elements) using his own method contained in the treatise Quadrature of the Parabola [1]. Consider first the following figures .

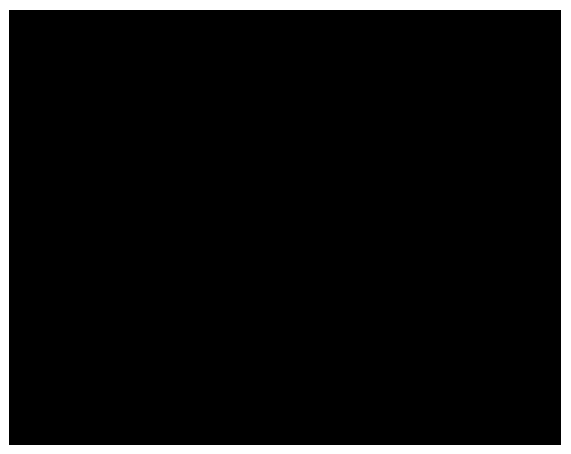

Figure 2.a

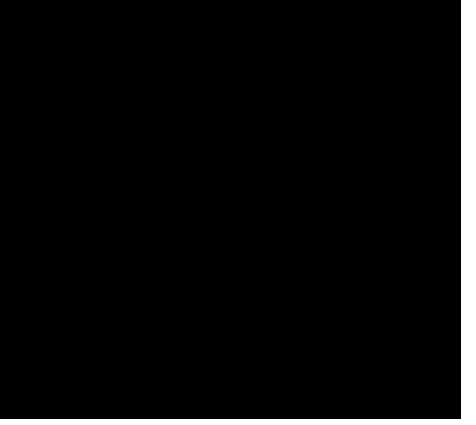

Figure 2.b

The construction and the preliminaries.

(1) Let $\gamma \beta \alpha$ be the parabolic segment and let $\beta \delta$ the diameter bisecting all chords parallel to the base $\gamma \alpha$ of the segment, thus $\delta$ is the midpoint of $\gamma \alpha$ (Figure 2.a).

(2) Obvious (it is proved as Proposition 18 in the Qudrature of the Parabola [1]) that the tangent at $\beta$ is parallel to $\gamma \alpha$.

(3) Take $\gamma \nu$ and $\alpha \mu$ parallel to $\delta \beta$. 


$$
\text { "klukovits" — 2005/2/17 — 18:26 — page } 404 \text { — \#8 }
$$

(4) The triangle $\gamma \beta \alpha$ is one half of the parallelogramme $\gamma \nu \mu \alpha$ (Proposition I. 41. Euclid Elements ).

(5) The triangle $\gamma \beta \alpha$ is greater than the half of the parabolic segment.

(6) Let $\delta_{1}$ and $\delta_{1}^{\prime}$ the midpoints of the chords $\gamma \beta$ and $\beta \alpha$ respectively, moreover let the lines $\delta_{1} \beta_{1}$ and $\delta_{1}^{\prime} \beta_{1}^{\prime}$ are parallel to $\delta \beta$, i.e. repeat the above procedure to the parabolic segments $\gamma \beta_{1} \beta$ and $\beta \beta_{1}^{\prime} \alpha$ (Figure 2.b).

Observe that the triangles (the area of them) $\gamma \beta_{1} \beta$ and $\beta \beta_{1}^{\prime} \alpha$ are together $\frac{1}{4}$ of the triangle $\gamma \beta \alpha$. Then by repeating again and again this process - similarly to that of Figure 2.a and Figure 2.b - we get (in finite number of steps) the geometric series

$$
\gamma \beta \alpha_{\triangle}+\frac{1}{4} \gamma \beta \alpha_{\triangle}+\frac{1}{16} \gamma \beta \alpha_{\triangle}+\ldots
$$

Remark. Although for a present day mathematician it is clear, that the area of the parabola segment $\gamma \alpha \beta$ is equal to the sum of this infinite series, for an ancient Greek mathematician such type of sum was impossible.

Archimedes found a brilliant method to avoid this type of infiniteness. It is based on the following propositions.

Proposition 23 (Quadrature of the Parabola [1]). Given a series of magnitudes, each of which is equal to four times the next in order, all the magnitudes and one third of the last added together will exceed the greatest by one-third.

Remark. The above proposition states in modern notations, that if $m_{1}, \ldots, m_{n}$ is a geometric progression with quotient $\frac{1}{4}$, then we have

$$
m_{1}+\ldots+m_{n}+\frac{1}{3} m_{n}=\frac{4}{3} m_{1}
$$

In the original proof Archimedes considered the case $n=5$ without really using this restriction. As it was general in the classical Greek mathematics where "finite number" always meant a concrete number - e.g. 3 for Euclid, or 5 for Archimedes -, not an arbitrary one, say $n$. We mention, that this proposition can also be proved using Proposition IX. 35. from Euclid Elements.

We can read in several treatises of Archimedes the following approximation method, which plays a crucial role in avoiding infiniteness. In modern terminology this reads as follows. 
The Archimedean Approximating Method [1]. A magnitude $\Sigma$ to be calculated is approximated from below by the sum $S_{n}$ of $n$ (positive) terms of a (convergent infinite) series, where we know that $\Sigma-S_{n}$ can, by the choice of $n$, be made less than any assigned magnitude $\varepsilon$, while the same applies to $a_{n}$. The calculation consists in finding a magnitude $K$, which for any value of $n$ satisfies the equality

$$
a_{1}+\ldots+a_{n}+R_{n}=K, \quad \text { where } R_{n}<a_{n} .
$$

Now we have $\Sigma=K$.

Proof. The Archimedean proof is a brilliant use of double reductio ad absurdum. He showed that both $\Sigma>K$ and $\Sigma<K$ are impossible. Namely, first suppose that $\Sigma>K$, and find an $n$ so

$$
\Sigma-S_{n}<\Sigma-K
$$

This implies $K<\Sigma$ contradicting (2).

Suppose now that $\Sigma<K$, and find $n$ so $a_{n}<K-\Sigma$. By (2) we have $K-S_{n}<$ $a_{n}<K-\Sigma$, i.e. $\Sigma<S_{n}$. This contradicts the assumptions on $\Sigma$ and $S_{n}$.

To prove the statement on the area of the parabolic segment $\gamma \alpha \beta$ consider the earlier mentioned

Proposition X. 1 (Euclid, Elements). Two unequal magnitudes being set out, if from the greater there be substracted a magnitude greater than its half, and from that which is left a magnitude greater than its half, and if this process be repeated continually, there will be left some magnitude which will be less than the lesser magnitude set out.

On the basis of this proposition the conditions of the approximating method are fulfilled. To finish the proof let denote by $\Sigma$ the area of the parabolic segment. If we take $m_{k}=\left(\frac{1}{4}\right)^{k-1} \gamma \beta \alpha \triangle$ for $k=1, \ldots, n$, we have by (2) that

$$
\gamma \beta \alpha_{\triangle}+\frac{1}{4} \gamma \beta \alpha_{\triangle}+\ldots+\left(\frac{1}{4}\right)^{n-1} \gamma \beta \alpha_{\triangle}+\frac{1}{3}\left(\frac{1}{4}\right)^{n-1} \gamma \beta \alpha_{\triangle}=\frac{4}{3} \gamma \beta \alpha_{\triangle} .
$$

While the assumptions of the approximation method satisfied, i.e., (3) can be used, we have that

as we stated.

$$
\Sigma=\frac{4}{3} \gamma \beta \alpha_{\triangle}
$$




$$
\text { "klukovits" — 2005/2/17 — 18:26 — page } 406 \text { — \#10 }
$$

Concluding methodological remark. For secondary school students or even for university students (except majoring mathematics) often there is no clear distinction between the heuristic argumentations and the rigorous proofs. We hope that the above classical example will help the teachers to make this distinction more clear for them.

Acknowledgements. The author is grateful to Zoltán Kovács, for his technical assistance in drawing the figures.

\section{References}

[1] E. J. Dijsterhuis, Archimedes, Munksgaard, Copenhagen, 1956.

[2] T. L. Heath, The thirteen books of Euclid's Elements, Cambridge University Press, 1956.

[3] M. Kline, Mathematical Thought from Ancient to Modern Times, Oxford University Press, 1972.

[4] B. L. van der Waerden, Science Awakening, Oxford University Press, 1961.

[5] B. L. van der Waerden, Geometry and Algebra in Ancient Civilizations, Springer-Verlag, 1983.

LAJOS KLUKOVITS

BOLYAI INSTITUTE

UNIVERSITY OF SZEGED

H-6720 SZEGED, ARADI VÉRTANÚK TERE 1

HUNGARY

E-mail: klukovits@math.u-szeged.hu

(Received July, 2004) 